\title{
POLÍTICA DE ATENCIÓN AL DESPLAZAMIENTO FORZADO EN COLOMBIA: ELEMENTOS PARA UNA EVALUACIÓN EN EL ÁMBITO LOCAL ${ }^{1}$
}

Katherine Flórez Pinilla

\begin{abstract}
Resumen
La migración forzada por la violencia generando pérdidas directamente afectados y a la sociedad en general. Esta doble afectación exige eficacia y oportunidad al Estado en la política pública creada para la satisfacción de demandas derivadas del fenómeno. Esta exigencia ha estado fuertemente cuestionada por organismos internacionales, por la Corte Constitucional colombiana y otros estamentos competentes, los cuales reclaman un seguimiento riguroso y periódico que permita evaluar y retroalimentar las intervenciones con el fin de conseguir la efectividad de las mismas. Antecedentes de evaluación coinciden en mediciones en el ámbito nacional, de acuerdo a agregados y aproximaciones estadísticas del fenómeno, dejando a un lado el seguimiento en el ámbito local, en los territorios directamente afectados. En tales circunstancias, el presente texto pretende proveer recomendaciones para la gestión y evaluación de la política pública para la atención del desplazamiento forzado en Santander.
\end{abstract}

\section{Palabras clave}

Desplazamiento forzado por la violencia en Colombia, evaluación de política pública, capacidad estatal.

\begin{abstract}
The violence forced migration generating losses directly affected and society at large. This double affectation demands effectiveness and timeliness to the State in public policy established to satisfy the claims arising from the phenomenon. This requirement has been strongly questioned by international bodies, by the Colombian Constitutional Court and other competent estates, which demand a rigorous and regular monitoring to assess and provide feedback interventions in order to achieve their effectiveness. Background measurements agree assessment nationally, according to aggregate and statistical approaches of the phenomenon, apart from monitoring at the local level, in the territories directly affected. In such circumstances, this text aims to provide recommendations for the management and evaluation of public policy to the attention of forced displacement in Santander.
\end{abstract}

\section{Key words}

forced displacement, Colombian public policy, national competence

1 Artículo producto de la investigación Evaluación de políticas de atención a población en situación de desplazamiento en el Área Metropolitana de Bucaramanga y municipio de Lebrija presentada por Katherine Flórez Pinilla y Vivianne Andrea Pinto Briceño para optar el título de Economistas en la Universidad Industrial de Santander, 2008.

2 Economista. Docente-Investigadora grupo de investigación Veritatem Universidad Santo Tomás, Seccional Bucaramanga. 


\section{Introducción}

La Organización de Naciones Unidas (ONU) define la migración forzada interna por la violencia como aquella situación según la cual "las personas se ven obligadas a huir de su hogar de residencia habitual como resultado o para evitar los efectos de un conflicto armado, de situaciones de violencia generalizada y de violaciones de los derechos humanos, que no han cruzado la frontera estatal internacionalmente reconocida" (1998). Dado que el fenómeno no constituye una acción voluntaria de los afectados, es deber del Estado gestar mecanismos suficientes para que dicho proceso pueda terminar lo más rápido posible, mitigar sus impactos, especialmente económicos y sociales, que permitan estabilizar a los afectados y garantizar la protección como ciudadano.

En Colombia, según datos de Acción Social, existen para el año 2009, 3.226.442 personas en situación de desplazamiento; en Santander la cifra asciende a 128.207 personas de las cuales el 70\% se encuentra en el Área Metropolitana de Bucaramanga (AMB). El registro oficial de población afectada existe en Colombia desde 1995, no obstante, aunque el fenómeno data de tiempo atrás, sólo se le ha brindado una respuesta sistemática y política desde 1997. En términos legales, en virtud de los derechos consagrados en la Constitución Política de Colombia, el Estado debe proteger a la población afectada, y de esta manera, está obligado a adoptar una política para afrontar esta situación. Aunque su implementación se ha llevado a cabo desde el ejecutivo, el fenómeno sigue en ascenso y los afectados no han conseguido la superación de su situación, evidenciándose ineficacia de los actuares públicos enfocados para este fin. Este artículo pretende exponer las razones por las cuales la política en cuestión no ha alcanzado sus pretensiones y las formas en que se puede encontrar salida favorable a esta situación. Se iniciará con un recorrido por el diseño de la política pública, su aplicación local y las dinámicas emergentes, se busca encontrar los factores limitantes del mismo y perspectivas de solución desde el ámbito local.

\section{Diseño y gestión de la política de atención al desplazamiento forzado en Colombia}

Evolución

La respuesta institucional al fenómeno se manifestó inicialmente por la expedición Ley 171 de 1994², aunque no brindó elementos de configuración de intervenciones sistemáticas para tratar los afectados, si se refirió a la posición del Estado con respecto al mismo; el artículo 17 prohibió eventos de desplazamiento forzado por la

3 Producto de la normatividad que protege a la población civil ante conflictos armados, los cuatro convenios de Ginebra de 1949, el protocolo II Adicional pactado en Colombia en 1977. 
violencia de la población civil ${ }^{4}$ y ante su inminente ocasión, exigió tomar medidas para que la población civil fuese acogida en condiciones satisfactorias de alojamiento, salubridad, higiene, seguridad y alimentación.

Sin embargo, sólo hasta la expedición de la Ley 387 de 1997 se gesta una directriz política para atender de manera integral el fenómeno, a saber: el Sistema Nacional Atención a Población Desplazada (SNAIPD). Esta política estuvo acompañada por el marco de cooperación gestado por la ONU y alimentada posteriormente con la expedición de los Principios Rectores para los Migrantes Internos ${ }^{5}$. Asimismo, dicho componente legislativo ${ }^{6}$ citado, retoma los contenidos del Documento CONPES 2804, así como del documento CONPES 2924 de 1997, los cuales establecen los criterios rectores para establecer dicha política pública.

Los productos legislativos, a los cuales se le puede atribuir el diseño de política en mención, fueron influenciados por diferentes elementos que configuran los momentos analíticos de todo accionar público, el primero referido al origen, determinado por el entorno político, expresado por la administración Samper, la cual reconoció la existencia del fenómeno y lo incluyó en su agenda de gobierno. En esta fase, en palabras de Lahera (2004), después de identificado el problema, los hacedores de políticas (policy maker) idean estrategias y cursos de acción para solucionarlo y planifican forma de llevarlas a cabo.

La planificación se expresó en la radicación de la Ley 387 de 1997 que creó el SNAIPD, el cual define el conjunto de entidades públicas, privadas y comunitarias a cargo de planes, programas y proyectos específicos tendientes a la atención integral para la población desplazada. Este sistema se propone brindar atención integral a los afectados; fortalecer el desarrollo, la promoción y protección de los derechos humanos y de las zonas expulsoras y receptoras; integrar los esfuerzos públicos y privados para la prevención y atención de las situaciones de desplazamiento; garantizar un manejo oportuno y eficiente de todos los recursos indispensables para la prevención y atención del fenómeno. Su accionar se configuró en torno a tres fases: la primera enfocada a la Prevención y Protección de factores que producen eventos de desplazamiento forzado; la segunda correspondiente a Atención Humanitaria que consta de acciones de socorro y atención a población desplazada para atender sus necesidades básicas o de subsistencia mínima una vez se haya presentado el

4 Artículo 17. (...) con excepción de aquellas que lo exijan para salvaguardar la seguridad de las personas civiles o razones militares imperiosas (...).

5 Los cuales recogen las obligaciones del Estado establecidas en los tratados internacionales en materia de los derechos humanos y el derecho internacional humanitario. Definen los derechos y garantías para proteger a las personas contra el desplazamiento y para su protección y asistencia durante estos eventos así como durante procesos de retorno y reintegración.

6 Según Ibáñez (2006), ésta legislación es considerada por los organismos internacionales como una de las más sólidas, elaboradas y avanzada en materia de atención. 
evento $^{7}$; y por último, la fase de Estabilización Socioeconómica, en la cual se deben promover acciones y medidas de mediano y largo plazo para generar condiciones de sostenibilidad económica y social para los afectados que permitan la cesación de su condición en el marco del retorno voluntario o el reasentamiento en otras zonas rurales o urbanas ${ }^{8}$.

Las organizaciones que integran el sistema se articulan alrededor del Consejo Nacional para la Atención Integral de la Población Desplazada (CNAIPD), órgano consultivo, encargado de formular las políticas y garantizar la asignación presupuestal de los programas que las entidades responsables tienen a su cargo. Entre sus labores se destaca la aprobación del Plan Nacional para la Atención Integral a Población Desplazada, como marco de actuación de las entidades, de acuerdo a las características y condiciones especiales de las zonas de expulsión y de recepción. El CNAIPD está conformado por los ministerios del ejecutivo, el ministerio público, el departamento nacional de planeación (DNP), Consejero presidencial para los derechos humanos, el comisionado de paz y el director de Acción Social (antigua RSS), este consejo es alimentado por el trabajo de cada unas de las mesas por fases de intervención y comités tanto a nivel nacional como de cada uno de los territorios descentralizados. A nivel local, según Decreto 2569 de 2000, los Alcaldes Municipales, Distritales y Gobernadores quedan encargados de la creación de los Comités de acuerdo a las fases de atención. Se establece además que las entidades estatales pueden concluir convenios con las ONG, Organizaciones No Gubernamentales, para brindar atención así como solicitar financiación a organismos internacionales.

Sin embargo, el funcionamiento del sistema demostró limitantes en la práctica, evidenciados por los reiterados llamados de la Corte Constitucional al ejecutivo y a las autoridades competentes, las sentencias expedidas ${ }^{9}$, en especial, la Sentencia T-025 de 2004, manifiesta que la situación que viven los desplazados representa un estado de cosas inconstitucional ${ }^{10}$, es decir, una violación masiva de los derechos constitucionales. La Corte consideró, basándose en las tutelas presentadas por los afectados ${ }^{11}$, que existe un desconocimiento masivo, generalizado y sistemático de los derechos de la población desplazada, debido a que el Estado, a través de acciones y omisiones en el diseño de la política, ha contribuido de una manera significativa

$7 \quad$ Artículo 15. Ley 387 de 1997. (...) socorrer, asistir y proteger a la población desplazada u atender sus necesidades de alimentación, aseo personal, manejo de abastecimientos, utensilios de cocina, atención médica y psicológica, transporte de emergencia y alojamiento transitorio en condiciones dignas.

8 Para profundizar sobre la oferta programática de programas de estabilización socioeconómica consultar Decreto 2569 de 2000 y Decreto 2007 de 2001.

9 Sentencias como la SU-1150 del 2000, la T-602 de 2003 y especialmente el pronunciamiento de la Sentencia T-025de 2004, la cual es la más relevante para analizar capacidad institucional del Estado.

10 Se declara cuando de manera insistente, se violan los derechos humanos a un grupo determinado de personas; cuando el elevado número de tutelas congestiona los despachos judiciales; y cuando la vulneración de los derechos se debe a factores estructurales, no sólo a la autoridad demandada.

11 Debido a 108 acciones de tutela interpuestas por 1150 familias de población desplazada se expide la Sentencia T- 025 
al desconocimiento de los derechos fundamentales de las personas en situación de desplazamiento.

Este argumento sintetiza los reclamos por parte de la Población en Situación de Desplazamiento (PSD), entre los cuales se destacan, la falta de orientación adecuada para tener acceso a los programas de atención y el sometimiento a un peregrinaje institucional, sin que se le dé una respuesta efectiva. Aunado a cierta ignorancia acerca de sus derechos, oferta institucional, procedimientos y requisitos para acceder a ella. En ocasiones, la respuesta de las entidades sólo se produce después de interpuesta la acción de tutela, ó se limita a informarles que no hay recursos suficientes para atender su solicitud y, que además, ésta se atenderá según el orden determinado por la entidad, sin que se les precise tiempo de espera.

Entre las principales solicitudes de los afectados por medio de las acciones de tutela se encuentran alrededor de la estabilización socioeconómica: materializar las ayudas para estabilización económica, recibir capacitación y apoyo para desarrollar proyectos productivos, vivienda, reubicación, acceso a educación para los hijos; protección a las tierras que fueron abandonadas; así como efectividad de la atención en salud y suministro de medicamentos recetados.

Esta declaratoria formal implicó un ajuste en las actuaciones de las autoridades encargadas de manera que lograra concordancia entre los compromisos adquiridos para cumplir los mandatos constitucionales y legales y los recursos asignados para asegurar el goce efectivo de los derechos ${ }^{12}$ de la población afectada. La situación de los afectados, según explica la Corte, no es imputable a una única autoridad, sino que obedece a un problema estructural que afecta a toda la política de atención diseñada por el Estado, en razón a la insuficiencia de recursos destinados a financiarla y a la precaria capacidad institucional para implementarla. Este problema institucional se ha expresado por la ausencia de indicadores y evaluaciones de desempeño, así como de impacto y seguimiento y verificación de las políticas por parte de los organismos del SNAIPD, lo cual constituye un requisito indispensable al momento de establecer la efectividad de las intervenciones y las fallas de las mismas.

En consecuencia, la Corte Constitucional ordenó, entre otras cosas que se diseñen y ejecuten políticas, planes y programas que garanticen de manera adecuada los derechos fundamentales; se solucionen las fallas de organización y de procedimiento que resultan violatorias de la Constitución; se diseñen mecanismo de evaluación y seguimiento de la política y se realicen los trámites administrativos y presupuestales que sean indispensables para superar la vulneración de los derechos. Y, por último,

12 Se hace referencia a los derechos catalogados como mínimos: derecho a la vida; a la dignidad e integridad física, psicológica y moral; derecho a la familia y su unidad; derecho a una subsistencia mínima como expresión del derecho fundamental al mínimo vital, derecho a la salud, a la protección frente a prácticas discriminatorias; derecho de los niños a la educación básica hasta los 15 años; Apoyo para el auto-sostenimiento por vía de la estabilización socioeconómica, derecho al retorno y al restablecimiento. 
que se apropien los recursos necesarios para garantizar la efectividad de tales derechos.

Por tal motivo se señala la importancia de adoptar un sistema de seguimiento de la gestión de las entidades que hacen parte del SNAIPD, que incluya un Sistema de Información confiable, debido a varias razones, en primer lugar, al problema del subregistro ${ }^{13}$, lo cual impide dimensionar el esfuerzo necesario para diseñar políticas, obstaculiza el control sobre las ayudas entregadas por otras agencias, y dificulta la evaluación del impacto de la ayuda entregada. En segundo lugar, los sistemas de registro no incluyen información acerca de las tierras abandonadas por los desplazados. Y por último, la información sobre cada desplazado no identifica las posibilidades de generación autónoma de ingresos en la zona de recepción, lo cual obstaculiza la implementación de las políticas de estabilización socioeconómica.

\section{Redefinición de la Política y búsqueda de soluciones desde lo local: Planes Integrales Únicos Territoriales y Fortalecimiento Institucional}

En respuesta a los pronunciamientos de la Corte, el diseño de la política cambió para fortalecer la institucionalidad y su capacidad de respuesta. El primer cambio evidenciado fue en el año 2005, según Decreto 250, que adopta por primera vez el PNAIPD, el cual establece un nuevo instrumento que garantice su ejecución a nivel territorial denominado Plan Integral Único (PIU). Sus funciones radican en ejercer la coordinación de las acciones de atención de acuerdo a las labores de los comités departamentales y municipales, construirlas, según una metodología de planeación estratégica participativa a corto, mediano y largo plazo alrededor de las tres fases de atención de la Ley 387.

En este marco, el trabajo de los Comités y las Mesas Territoriales se convierten en el principal insumo para el diseño y puesta en marcha del PIU: recoge el conjunto de estrategias, actividades, recursos y mecanismos de gestión que se formulan y concertan en el ámbito regional, para responder a las diversas demandas y necesidades de la población desplazada o en riesgo en el territorio, de acuerdo a las directrices de políticas nacionales del PNAIPD. Los planes del PIU se plasman en Planes Operativos por organización integrados por programas y proyectos.

Operacionalización de la Política en el Área Metropolitana de Bucaramanga y el municipio de Lebrija. Anterior a la puesta en práctica del PIU existía desde finales de 2000 en los municipios que conforman el AMB y Lebrija, un proceso de construcción de acciones hacia la solución de la problemática de la población desplazada de acuerdo al marco legal vigente ${ }^{14}$. Este proceso basado en el trabajo

13 En particular cuando se trata de desplazamientos menores, o individuales, en los que no se acude al Sistema para solicitar su inscripción

14 Se dice con Castro (2007) que Bucaramanga en el momento de expedición de la Ley 387 de 1.997, era el único municipio que contaba con una Oficina dedicada a la Atención denominada Consejería Municipal para la 
de comités territoriales ${ }^{15}$, se enmarcaba en el denominado Plan de Atención Integral a la Población Desplazada y Migrantes Económicos del AMB y Lebrija, con la participación de los diferentes actores locales del Sistema de Atención, la población desplazada y la sociedad civil.

En el año 2000, según Castro (2008), el Comité departamental plantea su trabajo de acuerdo a grupos de temáticas: salud, educación, vivienda, empleo y atención humanitaria, conformadas por las entidades competentes, las cuales “(...) presentaban informes en las sesiones del comité, pero no existía coordinación que permitiera optimizar esfuerzos y recursos lo cual permitió el desarrollo de actuaciones aisladas y desarticuladas". Ante esta situación, la Red de Solidaridad Social (hoy Acción Social), propone al Comité Departamental la necesidad de revisar el papel de estos Entes territoriales en el fortalecimiento del SNAIPD y recomienda la construcción de un Plan de Acción departamental, como instrumento de trabajo articulado. Para lograrlo, en el 2001, el Comité departamental, la Gobernación de Santander y la RSS construye un Diagnóstico y caracterización de la situación de la población afectada, con el propósito de sentar las bases para el diseño de una política sólida. En dicha caracterización se propone el Plan de Acción Departamental, el cual no tiene éxito en las sesiones de la Asamblea departamental y los Consejos Municipales, argumentándose escasa planeación y viabilidad.

En ese mismo año los Comités y la Arquidiócesis de Bucaramanga se unen con la Corporación Metropolitana de Planeación y Desarrollo de Bucaramanga (CORPLAN), en la denominada Alianza Interinstitucional dedicada al logro del bienestar de la población desplazada en los Municipios del AMB y Lebrija, tal proceso fue conocido como Macroproyecto, orientado hacia el tema de Generación de Ingresos, brindaba apoyo económico para capacitación laboral con los recursos dispuestos para el fortalecimiento de FOMIMYPES. Sin embargo, tal como afirma Castro (2008), la institucionalidad no conseguía aún coordinación e impacto de las políticas. Este hecho aunado a otros problemas de capacidad institucional sustentó un cúmulo importante de derechos de petición, acciones populares y de tutela que ubicaron también a Santander, en el marco de la Sentencia T-025 de 2004.

Ante esta coyuntura, organismos como ACNUR y otros organismos internacionales ${ }^{16}$ se integraron en el proceso de construcción de política local, coincidiendo este hecho con la promulgación del Decreto 250 de 2005, donde se decidieron canalizar los esfuerzos en torno al PIU para la atención bajo la responsabilidad de la Gobernación. Acompañada también por las organizaciones

Atención Integral a la Población Desplazada. Los demás municipios atendían a esta población a través de las Secretarias de Desarrollo Social en Floridablanca y Girón y de Gobierno en Piedecuesta.

15 Activados por el Decreto 489 del 11 de marzo de 1999.

16 Especialmente el apoyo de ACNUR, Organización Panamericana de la Salud -OPS, Oficina para la Atención de Asuntos Humanitarios -OCHA, Organización Internacional para las Migraciones -OIM y Consejería en Proyectos-PCS. 
de la población desplazada ${ }^{17}$ la integración de estas instancias se denominó Comité Técnico del PIU Departamental.

En una primera fase, el proceso de planeación del PIU se diseñó para el AMB y Lebrija en el periodo 2006-2008, contando con dos instancias: un Comité Directivo constituido por el Gobernador, Alcaldes de los municipios e Iglesia, y por otra, un Comité Técnico, integrado por la Gobernación, Acción Social, Defensoría del Pueblo, Adel-Corplan (inicialmente) y representantes de la Población desplazada. Todas éstas acompañadas por demás instancias que componen el SNAIPD tales como el Instituto Colombiano de Bienestar Familiar (ICBF); Instituto Colombiano de Desarrollo Rural (INCODER); Fondo Nacional de Vivienda; Servicio Nacional de Aprendizaje (SENA); Gobernación de Santander; Municipios de Bucaramanga, de Floridablanca, de Girón, de Lebrija, de Piedecuesta.

Todas las anteriores componen las mesas de Protección y Prevención, Atención Humanitaria de Emergencia, Estabilización Socioeconómica y la Mesa de Fortalecimiento a Organizaciones de Población Desplazada, las cuales participaron en el proceso de planeación del PIU mediante cuatro fases: caracterización, planeación estratégica y operativa, organización y aprobación. En la fase de planeación se definieron líneas estratégicas de intervención: Impacto Social, Integración, Participación y Gestión; Desempeño financiero y Capacidad Institucional, cada una de ellas con un horizonte de actuación de largo plazo 2007-2011, el cual garantizaría la continuidad y mejoramiento de las intervenciones.

\section{Propuesta de evaluación desde el ámbito local}

Si bien la evolución del diseño de la política da cuenta de un proceso fluctuante y complejo, de cambios ejercidos por presiones sociales. En Santander, se puede decir, que se ha avanzado en su aplicación, desde la implementación del PIU, especialmente en la formulación de accionares articulados y sistemáticos para atender la población desplazada asentada en el territorio ${ }^{18}$. No obstante, aunque la planeación participativa descentralizada introdujo mejorar en lo que a la planeación concierne, se hacía inminente la necesidad de formular otro componente esencial del diseño de la política: un sistema de evaluación.

En el presente trabajo se tomó como referencia el proceso del planeación del PIU en lo referido a la fase de estabilización socioeconómica, específicamente en dos componentes representativos de la misma: Atención en salud y Generación de

17 Convergencia Nacional de Organizaciones de Población Desplazada -CND, Asociación de Desplazados de Colombia-ANDESCOL y el sector Independiente.

18 Departamentos como Santander, Valle y Antioquia son los únicos que han evidenciado adelantos en la construcción y desarrollo de PIU's. 
ingresos, como ejemplo para iniciar un proceso de evaluación de la política que cumpla con las exigencias que el fenómeno requiere.

Según lo dispuesto en el artículo 17 de la Ley 387 de 1997, las medidas de estabilización socioeconómica deberán permitir el acceso directo de la población a servicios de salud, educación y vivienda y a la oferta social del gobierno en programas que permitan el autosostenimiento, relacionados con: proyectos productivos; Sistema Nacional de Reforma Agraria y del Desarrollo Rural Campesino; Fomento a la microempresa; capacitación y organización social; así como planes de empleo urbano y rural.

\section{Paso 1. Diagnóstico inicial salud y generación de ingresos}

Salud. La búsqueda del acceso y un mejor servicio para la PSD es la base esencial de los programas del área Salud, según la legislación y lo comprometido en el PNAIPD el Estado está encargado de garantizar el acceso de la población desplazada a los siguientes rubros: a) Sistema General de Seguridad Social en Salud (SGSSS), b) programas de vacunación y de c) asistencia psicosocial. De acuerdo a esto, las diferentes organizaciones competentes tienen un rol importante de operatividad de lo dispuesto dada su capacidad financiera y técnica para lograr las metas propuestas en los periodos establecidos. En Salud, la responsabilidad institucional depende de entidades como Acción Social, ICBF y las Secretarías de Salud Municipales y Departamental.

Para 2007 los Planes Operativos de tales entidades, aprobados durante el proceso de planeación, presentaron un total de 65 programas de atención en salud para el AMB y Lebrija, con una proyección de inversión de $\$ 6.013 .55$ millones de pesos. Para final del periodo (diciembre de 2007), de acuerdo a los resultados de la rendición de cuentas anual, se ejecutaron el $91 \%$ de los programas y se invirtieron el $77,46 \%$ de los recursos.

En términos de ejecución de programas, las entidades que no alcanzaron el cumplimiento de sus metas presupuestales fueron las Secretarias Municipales de Bucaramanga, Floridablanca y Lebrija. En términos de inversión, la Secretaria de Salud de Bucaramanga ejecutó el $66 \%$ de la inversión propuesta, la Secretaría de Salud de Santander (53\%) y el ICBF (63.56\%), por el contrario, Acción Social ejecutó el $100 \%$ de la inversión planeada.

Dentro del diagnóstico efectuado, se evidenció que las entidades encargadas de la prestación de servicios de salud para PSD poseen problemas de coordinación dentro del SNAIPD, en la medida que generan duplicidad de esfuerzos que no permiten una especialización apropiada de las instituciones para la atención adecuada; las entidades, en muchos casos, prestan los mismos servicios y dejan a un lado otros componentes. De igual forma, se enfrentan a problemas de asimetría en la información primaria de registro de población desplazada, este hecho distorsiona el 
análisis de efectividad interno (en la organización) y externo (frente al entorno) que puedan brindar las políticas públicas en este sector.

Asimismo, la atención en salud se enfrenta a los problemas propios del sistema de salud colombiano (inequidad y acceso limitado, entre otros) que obstaculizan aún más la eficacia de las políticas de atención a PSD. Finalmente, se puede afirmar que aunque existen altos rubros disponibles para inversión, no todos son finalmente ejecutados y no en todos los casos donde la inversión se da al $100 \%$, en términos operativos, las metas alcanzan los niveles esperados.

Generación de Ingresos. Para alcanzar la estabilización socioeconómica, se han propuesto, desde el PIU Santander, la línea Generación de Ingresos, en la cual las diferentes organizaciones y entes encargados, así como los directamente afectados, han concertado estrategias. Existen tres tipos de organizaciones: el primero corresponde a organizaciones de competencia nacional y con accionar territorial Incoder y Acción Social; el segundo, corresponde a organizaciones no gubernamentales de operadoras de recursos de Cooperación Internacional: Corporación Compromiso y la Alianza CAFICOM (Unión temporal de la Caja de Compensación CAJASAN, la Fundación Tecnológica FITEC y la Fundación CONPAZ).

De manera resumida las líneas de intervención establecidas para el año 2007, con el objetivo de brindar actividades productivas sostenibles y atender las necesidades económicas de la población en situación de desplazamiento fueron: a) Apoyo a emprendimientos, por medio de créditos y capital semilla para procesos productivos, acceso a programas de capacitación laboral y encadenamiento productivo, así como de asesoría empresarial, entre otros; b) Generación de empleo, lograr la vinculación de 300 personas en situación de desplazamiento en sectores estratégicos de la región es la principal meta que se señala para lograr la generación de empleo; y c) Apoyo a procesos de reubicación a PSD con vocación rural: ofrecer programas de subsidios para la adquisición de predios rurales y el respectivo acompañamiento institucional para que esta reubicación sea sostenible.

Los cursos de acción expresados en los planes operativos de cada organización en 2007 para cumplir el objetivo de Generación de Ingresos presentaron en la fase de planeación un total de 33 proyectos, con un costo global de $\$ 5.198 .17$ millones de pesos, de los cuales según la rendición de cuentas anual se ejecutaron 23 proyectos, equivalentes al $70 \%$ de los recursos ejecutados, \$ 3.950 .72 millones de pesos. Es de destacar que el $57 \%$ de los proyectos planeados estuvieron enfocados en la estrategia de Apoyo a Emprendimientos, el 21\% a Generación de Ingresos y el restante 21\% a la estrategia de Reubicación Rural Integral. Los cursos de acción ideados en la línea de emprendimientos consistían en su mayoría en programas de capacitación y fortalecimiento en proyectos productivos. 
Las entidades que menos cumplieron lo previsto fueron el Departamento y el Incoder, aunque los proyectos que estas últimas ejecutaron representaron cuatro (4) veces el valor de los proyectos ejecutados por los municipios en conjunto, los cuales presentan una mayor eficacia en términos de cumplimiento con lo planeado.

Figura 1. Financiación de Proyectos según Entidades de generación de ingresos en 2007

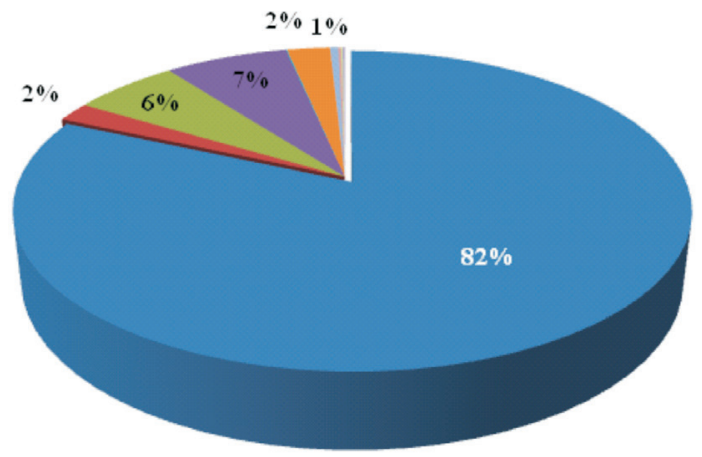

$$
\begin{aligned}
& \text { - Acción Social } \\
& \text { - SENA } \\
& \text { - Gobernación } \\
& \text { - INCODER } \\
& \text { - SENA } \\
& \text { - Bucaramanga } \\
& \text { - Floridablanca } \\
& \text { n Girón } \\
& \text { - Piedecuesta } \\
& \text { - Lebrija }
\end{aligned}
$$

Fuente: PIU (2007) y Cálculos propios

Con esta situación es importante destacar que la atención en generación de ingresos es crucial para garantizar a la recuperación su capacidad productiva y trascender a una etapa de estabilización socioeconómica que permita la cesación de su situación de desplazamiento. Por esta razón, es preocupante la ineficacia operativa de los proyectos planeados así como la insuficiente inversión por parte de las entidades territoriales (departamento y municipios) que no supera el 10\% de la inversión total ejecutada.

En este sentido, que aunque los programas de estabilización socioeconómica generan una carga financiera considerable para el gobierno, no se están gestando los mecanismos adecuados para generar resultados de impacto. De acuerdo con Ibáñez (2006),

(...) si no se diseñan de manera adecuada, se puede crear una población dependiente a la ayuda estatal. Para evitar esta dependencia, es necesario diseñar programas que ayuden a esta población a recuperar su capacidad productiva y fortalecer los programas de generación de ingresos; programas que son el componente más débil de la política actual (p. 10). 


\section{Paso 2. Diseño de indicadores de evaluación para la implementación local}

Para realizar la evaluación de impacto de los programas y proyectos desarrollados en el marco del PIU para atender a la población en situación de desplazamiento asentada en el AMB y Lebrija en el marco de su restitución de derechos, se diseñaron inicialmente 12 indicadores de acuerdo a los objetivos planteados para el objetivo de Actividades productivas sostenibles o Generación de Ingresos y 16 indicadores para el objetivo de Salud de acuerdo al direccionamiento estratégico 2007-2011.

Para su aplicación se realizó una prueba piloto que tenía como objetivo primario concientizar a los actores del sistema local en la importancia de la evaluación de sus accionares, así como la medición intermedia ${ }^{19}$ de los resultados en materia de Salud y Generación de Ingresos correspondientes a la fase de Estabilización socioeconómica establecida por Ley. Para su realización se contó con instrumentos metodológicos de conceptualización para abordar el tema de indicadores entre los funcionarios de las organizaciones competentes en cada una de las temáticas, así como socialización en el manejo del formato de captura ${ }^{20}$ de información y el entendimiento de cada indicador y su respectiva ficha técnica. La puesta en marcha del proceso de divulgación de los indicadores se realizó por medio de tres reuniones, la primera con el equipo de profesionales que conforma el PIU Santander así como el comité técnico, en las cuales se planteó en primera instancia la necesidad de diseñar indicadores externos a sus organizaciones que permitieran realizar seguimiento y evaluación al sistema de atención municipal enmarcado por el PIU.

La segunda reunión se realizó de manera independiente con cada uno de los representantes de las organizaciones que hacen parte de las mesas por temáticas, en la cual se explicó, la metodología de diseño de los indicadores para cada una de sus áreas, las particularidades de la medición en cada tema dadas sus características, por ejemplo, en Salud donde se discutía la importancia de seguir las mismas escalas de medida para que exista uniformidad en las cifras y no se preste a confusiones, así como la forma de hacer operacional una única base de datos con este mismo propósito.

Otro tema fundamental para discutir fue la importancia del elemento enfoque diferencial al momento de planear y sobretodo de medir cada uno de los accionares, cada uno de los indicadores incluía un elemento diferenciador por grupos especiales ${ }^{21}$ (niñez, grupos étnico, población con discapacidad y tercera edad, que tenía como eje transversal una perspectiva de género), que permitiera cuantificar la inclusión de

19 Evaluación intermedia debido a que se encuentra en marcha la política en cuestión.

20 Un ejemplo de los formatos de captura de información se puede apreciar en el sitio web DEL PIU Santander http://piusantander.gov.co/images/bateriaindicadores/ejemplo\%20indicador\%20salud.pdf

21 Según las recomendaciones de los autos de seguimiento de la Sentencia T 025 de 2004: Auto 177 de 2005, Auto 218 de 2006 y Auto 092 de 2008. 
estos grupos en la oferta de programas y así erradicar toda clase de discriminación o distinciones de carácter desfavorable en la población.

Después de esta segunda reunión se prosiguió a un tercer momento dedicado a la recolección de información relevante, utilizando el formato de captura aunado a los planes operativos por organización ${ }^{22}$. En este tercer momento se evidenciaron varias dificultades debido entre varias razones, a la movilidad de funcionarios públicos a la fecha de recepción de información dada la coyuntura de cambio de administraciones en las entidades territoriales, la falta de obligatoriedad para el cumplimiento de la fecha prevista, y la ausencia de sistematización de información relevante en cada organización.

Los resultados preliminares a la primera puesta en marcha de los indicadores pilotos están sujetos a la calidad, oportunidad y disponibilidad de la información suministrada por cada una de las organizaciones que configuran la oferta institucional de atención a población desplazada.

Es de destacar que el proceso de recolección de información estuvo marcado por diferentes hechos que permiten deducir varias características del sistema local: en primer lugar, la mayor parte de las organizaciones no poseen una base de datos interna clara y oportuna que permita realizar seguimiento periódico a sus actuaciones, por ejemplo, en salud, sólo los operadores de los servicios (EPS, IPS, ESES y Profamilia, entre otros) tienen los datos de atención pero éstos no son remitidos de manera efectiva a los controles centrales (en este caso Secretarías de salud municipal y departamental) con el fin de determinar el volumen de población atendida y sus características. Asimismo, no existe una línea base para planear de mejor forma, especialmente en el ámbito de salud, la medición de objetivos y metas que cambian periódicamente, y cada institución de atención en salud tiene su propia forma de recolección de datos, lo cual implica heterogeneidad de resultados, lo cual no permite una evaluación general, lo que genera sesgos dentro de los análisis que se puedan realizar.

En segundo lugar, las organizaciones en este primer momento, no demostraron puntualidad en la entrega de resultados al PIU, este argumento se puede atribuir a la inexistencia de mecanismos por parte de la institucionalidad local para incentivar la rendición de cuentas efectiva y satisfactoria. Este argumento también podría ser extensivo para las organizaciones operadoras de la ayuda proveniente de organismos de cooperación internacional vinculados.

22 Debido a que no se consiguió la presencia de todos los actores se realizó de manera descentralizada utilizado el Plan Operativo de cada organización con el objetivo de recoger la información incorporando las respectivas preguntas que alimentarían los indicadores al esquema propuesto. 
Preliminares en salud. Los resultados previos en materia de salud permitieron evidenciar que recibieron cobertura en valoración psicosocial para 2007 el 88\% de la población recepcionada en el AMB, sólo el 26,7\% de los niños en situación de desplazamiento poseen un estado nutricional adecuado, el 39\% de la población se encuentra afiliada a algún régimen de seguridad social en salud. La primera observación que se pudo obtener de la prueba piloto fue la falta de información por parte de las instituciones en términos de información operativa. Las instituciones tienden a preservar información contractual, y la información operativa no es medida, sino reflejada en términos de inversión y cantidad de programas o proyectos ejecutados. La falta de diferenciación entre estos aspectos no permite un conocimiento completo de las capacidades institucionales de atención a la población (Flórez, \& Briceño, 2008).

Preliminares en ingresos. Son insuficientes los resultados pilotos para poder emitir juicios sobre la conveniencia de la política de generación de ingresos adoptada al momento debido a la poca información manejada por cada organización, no obstante, se espera que con este proceso iniciado las entidades puedan interiorizar los cuestionarios realizados, así como cada formato de captura de información asociado a los indicadores, para alimentar el sistema de seguimiento de procesos y de información oportuna.

De la información generada en el 2007 se puede decir que, en términos de acceso a programas de emprendimiento, las organizaciones evidenciaron mayor cobertura de la propuesta en el plan, de 600 familias propuestas se brindó apoyo a 1324 en emprendimientos. Sólo se evidenciaron registros de las familias que accedieron a estos programas, más no se demostró un seguimiento de la sostenibilidad y sustentabilidad de estos programas de emprendimiento en la creación de unidades productivas o vinculación laboral. Es de destacar que el apoyo a emprendimiento tuvo un carácter individual, en el sentido que no incluyó el núcleo familiar como variable objetivo, esto se demuestra en el pobre resultado de $9.7 \%$ la cobertura familiar de los programas. A diferencia de la eficacia del cumplimiento de la meta 2007 para el caso de los programas de emprendimiento, sólo se cubrió el 39.3\% de las personas propuestas por la meta concertada del PIU, equivalente a 300 personas (Flórez, et., al. 2008).

\section{Balance de la política: objetivos vs. resultados}

A pesar de no tener los datos solicitados de manera completa de cada una de las organizaciones, con la información preliminar y el análisis de programas y proyectos pertenecientes a la intervención en la línea Generación de Ingresos, se pueden aceptar varias hipótesis al respecto. En cuanto al diseño, si bien la Ley 387 es clara en darle prioridad a los programas y proyectos que permitan el sostenimiento autónomo de las familias y su estabilización socioeconómica de manera permanente y digna, los cursos de acción seguidos para este propósito en el AMB y Lebrija aún 
se concentran en mayor medida en programas de capacitación temporales (Apoyo a Emprendimientos) que pueden contribuir por unos meses a mejorar de alguna forma la situación económica pero después de sus finalización las condiciones declinan significativamente.

Esta situación evidencia cierta debilidad en los cursos de acción para generar actividades productivas sostenibles, la cual de acuerdo con Ibáñez $(2006$, p. 10) radica principalmente en tres razones: la falta de escola ridad de la población afectada que incide en que la capacitación laboral no sea efectiva y dificulte posteriormente la vinculación a los mercados laborales y, por otro lado, la carencia de capital semilla para estimular la creación de microempresas, además los pocos programas de capital semilla no siempre contemplan ayudas paralelas que este capital sea asignado al consumo del hogar; en tercer lugar, los programas de capital semilla tienen un acompañamiento insuficiente durante el proceso de formulación e implementación del proyecto.

Dentro de los programas en el AMB y Lebrija vigentes en la primera fase del PIU, no se evidenció un componente claro de microcrédito sin barreras a la entrada para la población desplazada, si bien los programas vinculados a apoyo a emprendimientos y Generación de empleo incluyen capital semilla para proyectos productivos no poseen un mecanismo integral que permita el autosostenimiento de las familias, lo cual incide en que el capital otorgado no sea invertido efectivamente en lo planeado, sino en otros gastos familiares.

En la línea de Generación de empleo se encuentra que no existe información sobre la oferta de trabajo brindada por la PSD, si bien uno de los proyectos estaba encaminado a la creación de un sistema de información que contuviera la oferta y la demanda de trabajo, éste no se ha ejecutado, lo cual dificulta la planeación de cursos de acciones adecuados para la población. Entre los programas ejecutados para proveer empleo se encuentra que estos en su mayoría poseen el componente de capacitación donde resulta incierto su efectividad para vincular directamente a la población beneficiada a un empleo formal.

En cuanto a la atención en Salud, el principal problema radica en la presencia de sistemas de informaciones difusas y heterogéneas, no hay información confiable que permita la identificación completa de los casos, tratados así como las necesidades básicas insatisfechas. Parte de la atención de PSD no se radica en el sistema de registro adecuado, lo cual genera sesgos en los resultados finales. Estos sesgos generan indicadores con problemas de asimetría, inconsistencia y poca confiabilidad. La evaluación, por lo tanto, no es posible sin que existan sistemas de información completos.

Aunque se planean múltiples programas con el fin cumplir con las demandas de la PSD, su ejecución es aún insuficiente por lo cual queda comprometida la eficacia del sistema territorial, aunque no todas las organizaciones incumplan lo planeado. 


\section{Conclusiones}

La política de atención al desplazamiento forzado en Colombia pese su planeación desde hace más de una década ha demostrado avances desde su reestructuración en 2005 , en la cual le otorga mayor importancia a la planeación descentralizada y a la formulación de accionares públicos con enfoque de derechos. Sin embargo, esta política debe cumplir con todas sus fases, especialmente la evaluativa, para su efectiva realización y alcance de impactos esperados.

Un sistema de indicadores ajustado a la planeación descentralizada en el AMB y Lebrija y con criterios de equidad constituye la propuesta que hoy se implementa en territorio de estudio. Su implementación en sus inicios requirió de una sensibilización en el tema del desplazamiento y la importancia de la evaluación entre las entidades que conformar el sistema local.

El primer requisito que se debe cumplir para realizar una evaluación efectiva de la política es la caracterización de la población objetivo, su ubicación y sus necesidades con el fin de focalizar la oferta institucional hacia las necesidades objetivo, elemento que no se ha alcanzado dado las disparidades de bases de datos sobre registro. Los sistemas de información son la base esencial de las mediciones, por lo que es preciso solicitar información focalizada de los proyectos en ejecución a cada una de las entidades involucradas con el fin que permitan evitar sesgos y asimetrías dentro de las mismas.

La evaluación preliminar efectuada en el ámbito local en el año 2007, da cuenta que la atención de Salud podría brindarse eficazmente, una vez las organizaciones se incentiven a cumplir con las metas propuestas. Este incentivo se puede dar mediante la exposición pública de los resultados generados mediante los sistemas de evaluación de las políticas públicas. Es necesario hoy indagar los resultados que ha registrado el sistema desde empoderamiento en las entidades que se ha dado desde el año inmediatamente anterior y el presente.

En la oferta de estabilización socioeconómica, especialmente salud y generación de ingresos, se considera necesario en el AMB y Lebrija fortalecer los procesos de vinculación laboral adecuados a las capacidades y destrezas laborales de la población que permita su autosostenimiento y estabilización socioeconómica. Esta vinculación laboral se puede dar vía proyectos productivos autónomos, por medio de microcréditos y capital semilla que eliminen las barreras actuales para acceder a ellos. La vinculación también se puede dar por medio de programas de capacitación laboral que tengan entre sus objetivos realizar convenio interinstitucionales con el sector privado para proveer empleo y reducir los costos de búsqueda de vinculación laboral.

En necesario fomentar los procesos de reubicación de la población desplazada debido a que gran parte de ésta (52.41\%) manifiesta su deseo de reubicarse en un lugar 
digno y adecuado para garantizar gran parte de la estabilización socioeconómica y el goce efectivo de derechos. Más aún, se deben fomentar los procesos de reubicación a las personas que tienen vocación rural y que unido a la entrega de subsidios para adjudicación de predios por parte del INCODER, se realicen esfuerzos institucionales para brindar acompañamiento adecuado por medio de proyectos productivos.

\section{Referencias}

Acción social. Estadísticas sobre población desplazada en Colombia. Recuperado de Adel-Corplan (2005). Documento Caracterización del Desplazamiento en el Área Metropolitana de Bucaramanga y Lebrija y La Realidad Institucional. Abril de 2005

Castro, I (2008). El PIU: Propuesta Integral de Articulación de las Instituciones del SNAIPD. En Plan Integral Único de Santander de Atención a Población desplazada 2007-2011. (pp. 15-24). Santander, Colombia

Conpes 2429. Sistema Nacional de Atención Integral a la Población Desplazada por la Violencia

Conpes 2804 DE 1995. Programa Nacional de Atención Integral a la Población Desplazada por la Violencia

Conpes 3400. Metas y priorización de recursos presupuestales para atender a La población desplazada por la violencia en Colombia

Corte Constitucional (2004). Sentencia T-025 de 2004. Bogotá, Colombia.

http://www.accionsocial.gov.co/Estadisticas/publicacion\%20septiembre\%202009. htm noviembre 2009

Flórez, K., Briceño, V. A. (2008). Evaluación de políticas de atención a población en situación de desplazamiento en el Área Metropolitana de Bucaramanga y municipio de Lebrija. Tesis de grado. Universidad Industrial de Santander.

Ibáñez, A (2006). La estabilización Socioeconómica de la Población Desplazada. Fundación Ideas para la Paz, Working Papers, Noviembre de 2006. Tomado de:

www.ideaspaz.org/new_site/secciones/publicaciones/download_papers_fip/ working_papers_3.pdf

Lahera, E (2004). Economía Política de las Políticas Públicas. Artículo Electrónico recuperado de: http://www.ejournal.unam.mx/ecu/ecunam2/ecunam0204.pdf. en mayo de 2008

ONU (1998). Consejo Económico y Social de Naciones Unidas (1998). Principios

Rectores de los Desplazamientos Internos. Extracto del documento E/ CN.4/1998/53/Add.2, de 11 de febrero de 1998 
Plan Integral Único de Santander (2006). Documento Ejecutivo (2007) Planes Operativos 2007 por Municipios y por Organizaciones que conforman el Sistema Nacional de Atención Integral a Población Desplazada (2007). Direccionamiento Estratégico 2007-2011. PIU Santander Fase I desplazada 2007-2011

República de Colombia. Decreto 250 de 2005. Plan Nacional para la Atención Integral a la Población Desplazada por la Violencia. Ministerio del Interior y de Justicia, 7 de febrero de 2005

República de Colombia. Decreto 2569 de 2000. Por el cual se reglamenta parcialmente la Ley 387 de 1997 y se dictan otras disposiciones.

República de Colombia. Ley 171 de 1994. Por medio de la cual se aprueba el "Protocolo Adicional a los Convenios de Ginebra del 12 de agosto de 1949, relativo a la protección de las víctimas de los conflictos armados sin carácter internacional (Protocolo II)", hecho en Ginebra el 8 de junio de 1977.

República de Colombia. Ley 387 de 1997. Medidas para la prevención del desplazamiento forzado; la atención, protección, consolidación y estabilización socioeconómica de los desplazados internos por la violencia en Colombia. Congreso de Colombia, 18 de Julio de 1997. 